\title{
New frequency analyses of the multiperiodic $\delta$ Scuti variables CR Lyn and GG UMa
}

\author{
C.W. Robertson ${ }^{1}$, P. Van Cauteren ${ }^{2}$, P. Lampens ${ }^{3}$, E. García-Melendo ${ }^{4}$, R. \\ Groenendaels $^{5}$, J. Fox ${ }^{6}$, P. Wils ${ }^{7}$ \\ ${ }^{1}$ SETEC Observatory, Goddard, KS, USA, (cwr@pixius.net) \\ 2 Beersel Hills Observatory (BHO), Laarheidestraat 166, B-1650 Beersel, Belgium \\ (paulvancauteren@skynet.be) \\ ${ }^{3}$ Koninklijke Sterrenwacht van België, B-1180 Brussel, Belgium \\ (patricia.lampens@oma.be) \\ ${ }^{4}$ Esteve Duran Observatory (EDO), Montseny 46 - Urb. El Montanya, 08553 Seva, \\ Spain (duranobs@astrogea.org) \\ ${ }^{5}$ Dworp Observatory, B-1653 Dworp, Belgium (roger.groenendaels@skynet.be) \\ ${ }^{6}$ Summer fellow, Koninklijke Sterrenwacht van België \\ 7 Vereniging Voor Sterrenkunde, Belgium (patrick.wils@cronos.be)
}

\begin{abstract}
CCD photometric time-series observations of the $\delta$ Scuti stars CR Lyn and GG UMa reveal that both are multiperiodic pulsators, with at least three close, independent frequencies for CR Lyn and two frequencies in the case of GG UMa. In addition SAO 14823, another star in the field of GG UMa, is reported to be new multiperiodic variable as well.
\end{abstract}

\section{Introduction}

The Hipparcos mission discovered a large number of new variable stars including various $\delta$ Scuti stars (ESA 1997). Koen (2001) performed a statistical analysis of the HIPPARCOS epoch photometry in search of multiperiodicity among all stars which were classified as variable in the Hipparcos catalogue: periodic, unsolved and microvariables. Two of the multiperiodic stars detected among the periodic variables by Koen (2001), CR Lyn (= HIP 40651) and GG UMa (= HIP 45693), have been re-analysed using recent CCD time-series data collected at (mostly) private small observatories. Table 1 gives an overview of the instruments and photometry reduction packages used. 
Table 1: Instruments used for this study.

\begin{tabular}{lllll}
\hline Observer(s) & Observatory & Telescope & CCD camera & Software \\
\hline PVC, PL & BHO & 40-cm Newt. & SBIG ST10XMe & Mira AP \\
PVC & BHO & 25-cm Newt. & SBIG ST10XMe & Mira AP \\
PVC & Hoher List & 12.5-cm refr. & SBIG ST10XMe & Mira AP \\
CWR & SETEC & 30-cm Cass. & SBIG ST-8i & AIP4Win \\
EGM & EDO & 60-cm Cass. & SX Starlight & LAIA \\
RG & Dworp & 30-cm Newt. & Hisis44 & AIP4Win \\
\hline
\end{tabular}

\section{CR Lyn}

In 2002 and 2003, we collected a total of $9348 \mathrm{~V}$-measurements for CR Lyn during 254.4 hours in 42 nights. The observational log of the data is presented in Table 2. The last column (RMS) lists the nightly range of the variation in differential magnitude between the two comparison stars in the field. These are HIP $40632(V=8.73, B-V=0.48)$ and HIP $40562(V=8.91, B-V=$ $0.75)$. From the $B$ and $V$-data acquired at two observatories, a colour index of $B-V=0.35$ at maximum and $B-V=0.38$ at minimum was derived. Two individual light curves are illustrated in Fig. 1, from which the cycle-to-cycle variation of the amplitude is clearly seen. The maximum total $V$-amplitude is about $0.13 \mathrm{mag}$.

CR Lyn (= HIP 40651, V=7.65 mag, spectral type F0) was found by Koen (2001) to show frequencies of 7.59057 and $7.29149 \mathrm{c} / \mathrm{d}$ and amplitudes of 0.031 and $0.021 \mathrm{mag}$, respectively, from the HIPPARCOS photometry (see Table 2 in his work). A frequency analysis of the new $V$-data set using Period04 (Lenz \& Breger 2005) shows the same two frequencies. However, in contrast to Koen's results, we found two equally dominant frequencies (i.e., within their probable errors). This can be seen in Table 3, where we list the frequencies and the linear combinations of these frequencies, together with their semi-amplitudes and phases, in order of decreasing amplitude. The signal-to-noise ratio was calculated after prewhitening for all the frequencies. Formal uncertainties are given between parentheses. The last column indicates the reduction in total variance accounted for by this frequency.

Since the amplitude in the $H_{p}$ passband is not exactly the same as the amplitude in the $V$ passband ( $H_{p}$ broader), a direct comparison cannot be made. However, the ratio of both amplitudes can be compared and we suggest that either, the amplitude of one of the major frequencies changed over several years, or, an additional frequency is affecting the observed amplitude ratio. We 
Table 2: Observational log for CR Lyn.

\begin{tabular}{lccrccc}
\hline Observers & Filter & $\begin{array}{c}\text { Timespan } \\
\text { JD-2450000) }\end{array}$ & $\begin{array}{r}\text { Nr of } \\
\text { nights }\end{array}$ & $\begin{array}{c}\text { Nr of } \\
\text { hours }\end{array}$ & $\begin{array}{c}\text { Nr of } \\
\text { data }\end{array}$ & $\begin{array}{c}\text { RMS } \\
\text { (mag) }\end{array}$ \\
\hline RG & $V$ & $2257-2342$ & 8 & 37.0 & 2082 & $0.009-0.023$ \\
CWR & $V$ & $2671-2739$ & 11 & 73.9 & 1882 & $0.009-0.014$ \\
PVC, PL & $V$ & $2681-2745$ & 12 & 65.7 & 3239 & $0.005-0.013$ \\
EGM & $V$ & $2709-2753$ & 12 & 77.8 & 2145 & $0.005-0.009$ \\
PVC, PL & $B$ & $2681-2689$ & 4 & 24.6 & 352 & $0.007-0.013$ \\
CWR & $B$ & $2708-2739$ & 6 & 34.6 & 1930 & $0.008-0.022$ \\
PVC, PL & $R$ & $2681-2689$ & 3 & 15.9 & 290 & $0.007-0.015$ \\
CWR & $R$ & $2708-2739$ & 6 & 34.7 & 1934 & $0.008-0.020$ \\
\hline
\end{tabular}

Table 3: Observed frequencies for CR Lyn ( $V$-data).

\begin{tabular}{lcccrcc}
\hline Id. & $\begin{array}{c}\text { Freq. } \\
(\mathrm{c} / \mathrm{d})\end{array}$ & $\begin{array}{c}\text { Semi-ampl. } \\
(\mathrm{mag})\end{array}$ & $\begin{array}{c}\text { Phase } \\
(\mathrm{cycles})\end{array}$ & $\mathrm{S} / \mathrm{N}$ & $\begin{array}{c}\text { Resid. } \\
(\mathrm{mag})\end{array}$ & $\begin{array}{c}\text { Reduct. } \\
\text { var. }\end{array}$ \\
\hline$f_{1}$ & $7.29172(1)$ & $0.0225(2)$ & $0.628(2)$ & 28.8 & 0.020 & $24 \%$ \\
$f_{2}$ & $7.59056(1)$ & $0.0196(2)$ & $0.398(2)$ & 25.5 & 0.016 & $39 \%$ \\
$f_{3}$ & $7.31341(2)$ & $0.0104(2)$ & $1.000(3)$ & 13.3 & 0.015 & $45 \%$ \\
$f_{1}+f_{2}$ & $14.88228(2)$ & $0.0024(2)$ & $0.295(14)$ & 5.5 & 0.014 & $46 \%$ \\
$2 f_{2}$ & $15.18112(2)$ & $0.0023(2)$ & $0.242(15)$ & 5.2 & 0.014 & $46 \%$ \\
$f_{2}+f_{3}$ & $14.90397(3)$ & $0.0018(2)$ & $0.787(19)$ & 4.1 & 0.014 & $46 \%$ \\
\hline
\end{tabular}

prewhitened the data for the two main frequencies and searched for yet another one. Indeed, a third frequency, $f_{3}$, was detected very close to $f_{1}$ in the power spectrum obtained after prewhitening (Fig. 2). The day ${ }^{-1}$ aliases of $f_{3}$ are also visible. The difference between $f_{3}$ and $f_{1}$ is only $0.02 \mathrm{c} / \mathrm{d}$, but well above the theoretical resolution of $1.5 / \Delta T=0.003 \mathrm{c} / \mathrm{d}$ for our data set.

Because of the much smaller time span, no frequency searches were performed using the $B$ and $R$-data. Instead, amplitudes and phases were calculated for the frequencies we derived from the $V$-data set. These are listed in Table 4. 

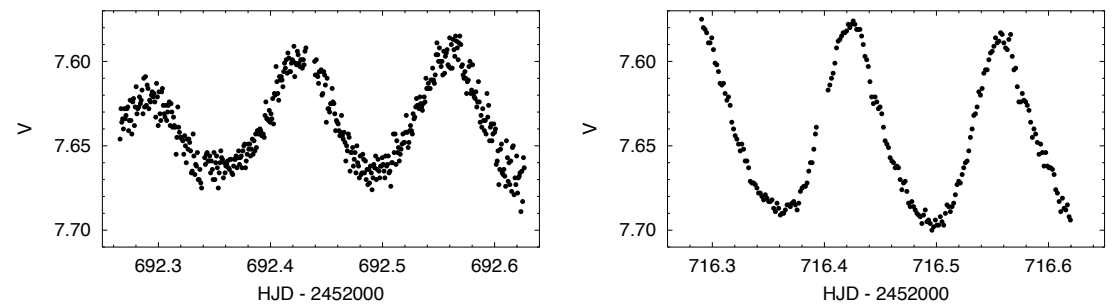

Figure 1: $V$ light curves of CR Lyn for two nights. Data are from BHO (left) and EDO (right panel).

Table 4: Semi-amplitudes and phases for CR Lyn in the filters $B$ and $R$.

\begin{tabular}{lcccc}
\hline Id & $\begin{array}{c}\text { Semi-ampl. } \\
B(\mathrm{mag})\end{array}$ & $\begin{array}{c}\text { Phase } B \\
\text { (cycles) }\end{array}$ & $\begin{array}{c}\text { Semi-ampl. } \\
R \text { (mag) }\end{array}$ & $\begin{array}{c}\text { Phase } R \\
\text { (cycles) }\end{array}$ \\
\hline$f_{1}$ & $0.0336(6)$ & $0.330(3)$ & $0.0249(5)$ & $0.748(4)$ \\
$f_{2}$ & $0.0265(6)$ & $0.476(4)$ & $0.0194(5)$ & $0.662(5)$ \\
$f_{3}$ & $0.0126(7)$ & $0.166(8)$ & $0.0104(6)$ & $0.200(10)$ \\
$f_{1}+f_{2}$ & $0.0019(5)$ & $0.203(43)$ & $0.0024(5)$ & $0.855(32)$ \\
$2 f_{2}$ & $0.0013(6)$ & $0.318(67)$ & $0.0020(5)$ & $0.637(44)$ \\
$f_{2}+f_{3}$ & $0.0015(6)$ & $0.205(60)$ & $0.0013(6)$ & $0.371(73)$ \\
\hline
\end{tabular}

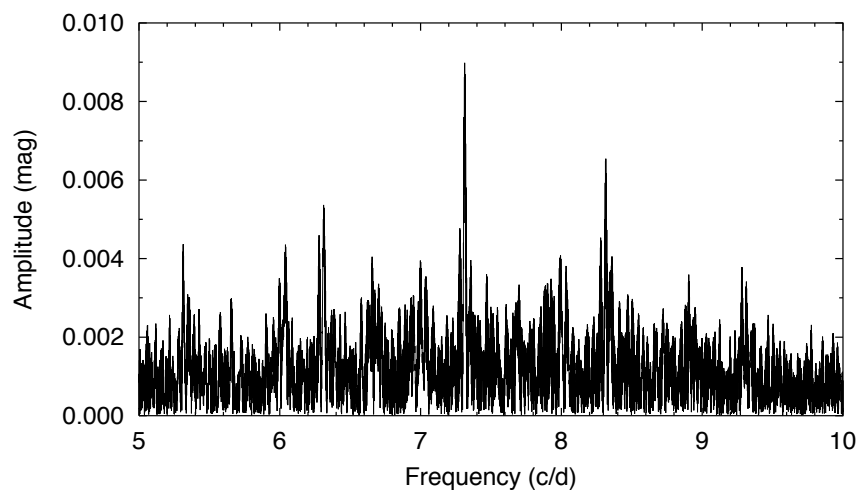

Figure 2: Frequency spectrum of CR Lyn after prewhitening $f_{1}$ and $f_{2}$.

\section{GG UMa}

We collected a total of $3385 V$-measurements for GG UMa during 75.2 hours in 15 nights during the winter 2003-2004. The observational log is presented 
Table 5: Observational log for GG UMa.

\begin{tabular}{lccrccc}
\hline Observers & Filter & $\begin{array}{c}\text { Timespan } \\
\text { (JD-2450000) }\end{array}$ & $\begin{array}{r}\text { No of } \\
\text { nights }\end{array}$ & $\begin{array}{c}\text { No of } \\
\text { hours }\end{array}$ & $\begin{array}{c}\text { No of } \\
\text { data }\end{array}$ & $\begin{array}{c}\text { RMS } \\
\text { (mag) }\end{array}$ \\
\hline PVC, PL & $V$ & $2992-3120$ & 9 & 38.1 & 2258 & $0.016-0.030$ \\
CWR & $V$ & $3018-3095$ & 6 & 37.0 & 1960 & $0.011-0.029$ \\
PVC, PL & $B$ & $3095-3097$ & 2 & 9.4 & 212 & $0.020-0.025$ \\
\hline
\end{tabular}

Table 6: Observed frequencies for GG UMa.

\begin{tabular}{ccccccc}
\hline Id. & $\begin{array}{c}\text { Freq. } \\
(\mathrm{c} / \mathrm{d})\end{array}$ & $\begin{array}{c}\text { Semi-ampl. } \\
(\mathrm{mag})\end{array}$ & $\begin{array}{c}\text { Phase } \\
(\text { cycles })\end{array}$ & $\mathrm{S} / \mathrm{N}$ & $\begin{array}{c}\text { Residual } \\
(\mathrm{mag})\end{array}$ & $\begin{array}{c}\text { Reduction } \\
\text { var. }\end{array}$ \\
\hline$f_{1}$ & $7.41526(6)$ & $0.0319(4)$ & $0.108(2)$ & 18.8 & 0.021 & $36 \%$ \\
$f_{2}$ & $7.83527(11)$ & $0.0179(4)$ & $0.969(4)$ & 10.4 & 0.017 & $48 \%$ \\
\hline
\end{tabular}

in Table 5. The $B$-filter data collected at $\mathrm{BHO}$ were not used in the frequency analysis. They were used to derive an average $B-V$ for GG UMa of 0.43 . The following comparison stars were used: SAO 14841 (= GSC 4138-0077, $V=9.52, B-V=0.61)$ and GSC 4138-0061 $(V=11.65, B-V=0.91)$. Two light curves are shown in Fig. 3. Koen (2001) derived two frequencies, 7.41615 and $7.83449 \mathrm{c} / \mathrm{d}$, with the respective amplitudes of 0.029 and 0.018 mag for GG UMa (= HIP 45693, V=8.60 mag, spectral type F5) from the HiPPARCOS mission. We confirm both from the frequency analysis of our $V$ data set. The frequencies with their corresponding semi-amplitudes and phases are listed in Table 6. As before, we also list the signal-to-noise ratio (after prewhitening), the residual RMS and the fraction of the reduction in total variance.

Although the residuals after prewhitening for both frequencies are still large $(0.017 \mathrm{mag}$; note that because the check star was relatively faint, the standard deviation of its differential magnitude is larger as well), none of the additional candidate frequencies found so far had a signal-to-noise ratio higher than 4. Therefore, they may be spurious. We conclude that, given the much shorter time span of this $V$-data set (only 128 days), any additional frequency cannot be resolved at this stage. GG UMa may be an evolved star as the F5 spectral type is cool for a main-sequence $\delta$ Scuti variable. 

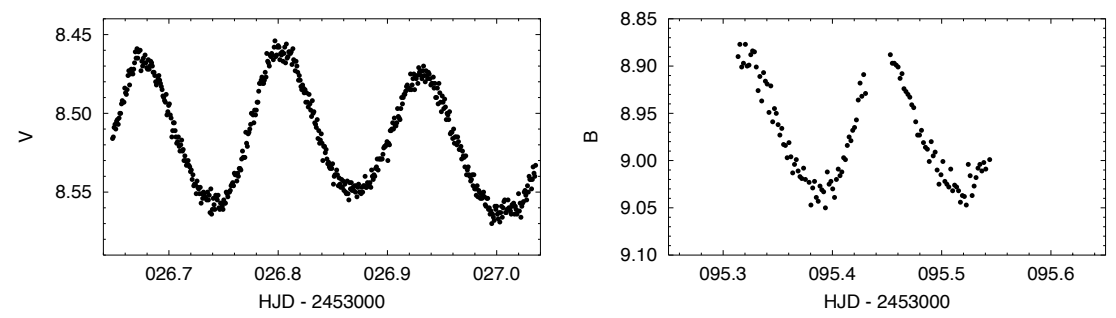

Figure 3: Light curves of GG UMa for two nights. $V$-data are from SETEC (left) and $B$-data from $\mathrm{BHO}$ (right panel).

\section{SAO 14823}

We further report the detection of short-period variability in the light curves of SAO 14823 (= GSC 4138-1569), which is located in the same field as GG UMa (see Fig. 4). Our data reveal a frequency of $23.7 \mathrm{c} / \mathrm{d}$ with a total amplitude in $V$ of almost $0.04 \mathrm{mag}$ as well as slow variations on a timescale of several days and with similar amplitude. Being of spectral type A0, this star may well be another $\delta$ Scuti star. Another phenomenon than pulsation might be needed to explain the existence of the slow variations found. However, our data are presently quite insufficient to characterise this longer-term variability.

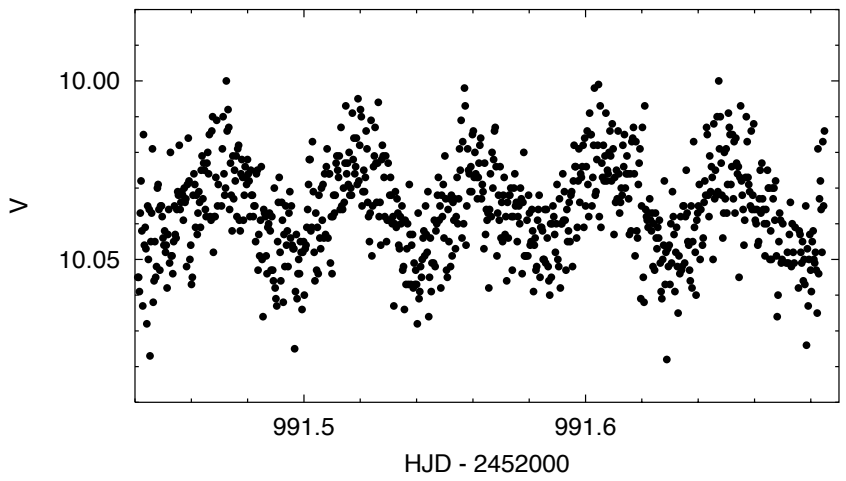

Figure 4: Light curve of SAO 14823 from BHO. 


\section{Conclusions}

New time-series CCD photometry was acquired for two $\delta$ Scuti variable stars found to be multiperidiodic by Koen (2001). In the case of CR Lyn, the total time span of our data equals almost 500 days and we were able to identify three independent frequencies, two of which $\left(f_{1}\right.$ and $\left.f_{3}\right)$ are very close to each other. In addition, an inverted amplitude ratio for the frequencies $f_{1}$ and $f_{2}$ was detected with respect to Koen's (2001) findings based on HipPARCos data. In the case of GG UMa, we confirm the previous frequency analysis and are unable to detect more than two frequencies, mainly due to the much shorter timespan of our data set (based on one season only) We also report the detection of very short-period variations as well as a longer-term variability in the light curves of SAO 14823, located in the field of GG UMa.

Acknowledgments. The Belgian data have been acquired with equipment purchased thanks to a research fund financed by the Belgian National Lottery (1999). EGM thanks Joan A. Cano and Rafael Barberá for developing the LAIA software. PL acknowledges support from the Fund for Scientific Research (FWO) - Flanders (Belgium) through project G.0178.02. PVC thanks Dr. K. Reif of the Hoher List Observatory, Universitätsternwarte Bonn, for the allocated telescope time. JF acknowledges a fellowship from the Belgian Science Policy Office during August 2004. This research made use of the Simbad and VizieR databases operated by the Centre de Données Astronomiques, Strasbourg, France.

\section{References}

Koen, C. 2001, MNRAS, 321, 44

Lenz, P., \& Breger, M. 2005, CoAst, 146, 53

ESA 1997, The HipparCOS and TYCHO catalogues, ESA SP-1200 